\title{
ORIGINAL
} ARTICLES

\section{Using a Delphi Technique to Define a Feedback Culture in Graduate Medical Education}

\author{
Cristen P. Page, MD, MPH; Hannah M. Baker, MPH; Linda Myerholtz, PhD
}

\begin{abstract}
BACKGROUND AND OBJECTIVES: Experts in medical education hypothesize that programs with a robust culture of feedback foster learning and growth for learners and educators, yet the literature shows no consensus for what defines a feedback culture in graduate medical education.

METHODS: Using a two-round, modified Delphi technique in summer and fall of 2019, the authors asked a panel of experts to identify essential elements to a feedback culture. The research team compiled a list of experts and a list of 29 descriptors of a highly functioning feedback culture. Experts rated the items as an essential, compatible, or not important aspect of a highly functioning culture of feedback. Researchers set a minimum threshold of $80 \%$ agreement and used comments from panelists to revise elements that did not meet agreement during round one. Experts then rerated the elements using information on their initial ratings, aggregate panelist ratings, and comments from all panelists.
\end{abstract}

RESULTS: The response rates from our panel of experts were $68 \%(17 / 25)$ for round one and $88 \%(15 / 17)$ for round two. Seventeen elements were rated as essential to a feedback culture.

CONCLUSIONS: An expert panel endorsed essential elements that can be used to assess feedback culture in graduate medical education programs.

(Fam Med. 2021;53(6):433-42.)

doi: 10.22454/FamMed.2021.600416

eedback is an important part of the learning process, and has been shown to affect the clinical performance of physicians. ${ }^{1}$ Research focus has shifted from the needs and behaviors of feedback providers to focus more on needs and perceptions of feedback receivers. Multiple studies explore resident perceptions and barriers to cultivating a positive culture of feedback. ${ }^{2,3}$ Much of the literature discusses feedback culture of programs as enabling or inhibiting feedback, and some literature provides the broad recommendation to "improve the overall feedback culture."4 Some have attempted to collect or provide definitions of feedback culture. For example, through focus groups and interviews, Ramani et al found that educators and learners define feedback as "one that communicated clear institutional expectations promoting regular two-way feedback conversations." ${ }^{\prime 5}$ London et al describe a strong feedback culture as "one where individuals continuously receive, solicit, and use formal and informal feedback to improve their job performance." J.C. Archer describes a feedback culture as one in which "feedback is embedded implicitly and explicitly in all activities and in which students feedback to teachers as well as teachers to students."7

Similarly, literature provides recommended ways to facilitate a feedback culture within an organization (some of which are specific to graduate medical education [GME]). Ramani et al developed 12 tips to promote a feedback culture within medical education, focusing their tips on sociocultural elements that include the relationships between giver and receiver, as well as institutional context. ${ }^{8}$ Kraut and colleagues suggest six strategies for facilitating an effective feedback culture in medical education, ranging from specific operationalized suggestions, for example "create daily triggers for feedback" to broader programmatic goals, such as "differentiate feedback from assessment." London et al provide guidance on organizational practices and interventions with three overarching themes related to feedback: (1) enhancing the quality, (2) emphasizing its importance within

From the School of Medicine (Dr Page), and Department of Family Medicine (Dr Myerholtz and Mrs Baker), University of North Carolina at Chapel Hill. 
the organization, and (3) providing support for using it. ${ }^{6}$ Yet, with each of these publications and suggestions for facilitating a feedback culture, we could not find consensus in the literature on either what defines a feedback culture or what steps a program can take to facilitate such a culture.

Using case study methodology similar to Bodenheimer et al, ${ }^{9}$ we gleaned learnings from over 50 graduate medical education programs across the United States, most of which are family medicine residencies, to implement and sustain direct observation feedback tools. ${ }^{10-12}$ Through sustained work with 27 residency teaching practices in a regional primary care residency collaborative active in three states, Donahue, Newton, Page and colleagues collected data showing wide variation in clinical quality and utilization. ${ }^{13,14}$ Through this combined work, Page et al identified variation in program needs and general readiness to adopt feedback practices, and also discerned a possible association between a culture of continuous quality improvement and programs with enhanced elements of feedback practices. There are tools designed to assess cultural elements of continuous quality improvement in academic clinical practice, such as the Patient Centered Medical Home Assessment ${ }^{15}$ used in the Building Blocks of High Performing Primary Care, ${ }^{9}$ as well as tools that help to measure hospital organizational culture as it relates to improving the quality of cardiovascular care. ${ }^{16}$ Yet, there is no such assessment tool to assess a culture of feedback in family medicine residency programs or in GME in general. Given family medicine residency training includes multiple clinical training environments and microcultures in many specialties, we must start with the need to define a culture of feedback in the broader context of GME.

The purpose of this study was to establish expert agreement on the key required elements that define a robust feedback culture in GME. By using the Delphi technique, we harnessed the expertise of individuals in the field who have contributed to the considerable body of literature related to the topic of feedback and feedback culture.

\section{Methods}

To identify key elements of a feedback culture in GME programs, we used the modified Delphi technique as described by Humphrey-Murto et al. ${ }^{17}$ The Delphi method uses a panel of experts to anonymously come to consensus on a topic while minimizing the influence of group thinking processes.

\section{Item Identification}

We searched the literature for evidence around defining a feedback culture, focusing on literature that relates to graduate medical education. This review of the literature allowed us to identify previously defined components of a feedback culture from 10 different peer-reviewed journal articles, and organize them into various dimensions. We then reviewed each dimension and developed one single definition that accurately captured each. Throughout this process, we attempted to maintain original wording from the published literature as much as possible. Upon reviewing the definitions, it became clear that they naturally separated into two categories: (1) elements of a feedback culture, and (2) ways to operationalize a feedback culture. With these two categories in mind, we developed the first category comprising 15 elements, and the second category comprising 14 elements (Table 1).

\section{Expert Panel}

We identified potential experts for our panel by reviewing the literature for existing, published definitions of a culture of feedback. We did not include published year as a limitation in our search, but the publications we found dated back as early as 2002. Though our search was not exhaustive, we felt that we had compiled a comprehensive list of published definitions from approximately 10 publications. From those publications, we invited the first, second, and senior authors to participate in the study as an expert panelist. We also asked these experts to nominate other possible experts. We invited potential panelists to participate in the study via email in May 2019. We did not offer incentives to panel experts. Our initial expert panel consisted of 30 individuals, all of whom had published scholarly articles on providing feedback and/or on feedback culture.

\section{Delphi Survey Process}

To minimize burden on our experts, we made an a priori decision to use a two-round Delphi process. The list of elements gleaned from the literature review was sent to our panel of experts using Qualtrics software (Qualtrics, Provo, UT), an online survey platform. We asked panelists to rate each element with the following ratings: "essential to a feedback culture," "compatible with a feedback culture," or "not important to a feedback culture." We instructed them to consider their responses in the context of a graduate medical education setting. We also invited the experts to provide open-ended comments regarding each element. Panelist responses were confidential.

Using guidelines from HumphreyMurto et al, where $80 \%$ is the highest minimum threshold suggested for use in Delphi studies, ${ }^{17}$ we set a minimum threshold of $80 \%$ agreement; elements that had $80 \%$ or higher agreement on the rating "essential to a feedback culture" were retained. Elements that had $80 \%$ or higher agreement on the rating "not important to a feedback culture" were discarded.

The research team consisted of two family medicine faculty and a research associate, all of whom have experience in the Delphi technique, and have previously been involved in, and published on, research related to giving and receiving feedback in graduate medical education. ${ }^{10-12}$ As a team, the researchers reviewed 
Table 1: Expert Panelist Ratings of Feedback Culture Elements for Round One and Two of the Delphi Study

\begin{tabular}{|c|c|c|c|c|c|c|c|c|c|}
\hline \multicolumn{5}{|c|}{ Round One } & \multicolumn{5}{|c|}{ Round Two } \\
\hline & $\begin{array}{l}\text { Essential } \\
\text { to a } \\
\text { Feedback } \\
\text { Culture }\end{array}$ & $\begin{array}{l}\text { Compatible } \\
\text { With a } \\
\text { Feedback } \\
\text { Culture }\end{array}$ & $\begin{array}{c}\text { Not } \\
\text { Important } \\
\text { to a } \\
\text { Feedback } \\
\text { Culture }\end{array}$ & $\begin{array}{c}\text { Total } \\
\mathbf{N}\end{array}$ & Revised Version & $\begin{array}{l}\text { Essential } \\
\text { to a } \\
\text { Feedback } \\
\text { Culture }\end{array}$ & $\begin{array}{l}\text { Compatible } \\
\text { With a } \\
\text { Feedback } \\
\text { Culture }\end{array}$ & $\begin{array}{c}\text { Not } \\
\text { Important } \\
\text { to a } \\
\text { Feedback } \\
\text { Culture }\end{array}$ & $\begin{array}{c}\text { Total } \\
\mathbf{N}\end{array}$ \\
\hline \multicolumn{10}{|c|}{ Essential Elements of a Feedback Culture } \\
\hline $\begin{array}{l}\text { 1. Faculty } \\
\text { serve as role } \\
\text { models about } \\
\text { how to seek, } \\
\text { receive, and } \\
\text { use feedback. }\end{array}$ & $100 \%$ & $0 \%$ & $0 \%$ & 17 & & & & & \\
\hline $\begin{array}{l}\text { 2. Everyone } \\
\text { receives } \\
\text { feedback } \\
\text { (ie, it's not } \\
\text { voluntary). }\end{array}$ & $82 \%$ & $12 \%$ & $6 \%$ & 17 & & & & & \\
\hline $\begin{array}{l}\text { 3. Learners act } \\
\text { as drivers of } \\
\text { change in their } \\
\text { local feedback } \\
\text { cultures. }\end{array}$ & $38 \%$ & $56 \%$ & $6 \%$ & 16 & $\begin{array}{l}\text { 3. Learners are } \\
\text { empowered to } \\
\text { improve their } \\
\text { local feedback } \\
\text { cultures. }\end{array}$ & $53 \%$ & $47 \%$ & $0 \%$ & 15 \\
\hline $\begin{array}{l}\text { 4. Longitudinal } \\
\text { relationships } \\
\text { between faculty } \\
\text { members and } \\
\text { learners exist. }\end{array}$ & $47 \%$ & $47 \%$ & $6 \%$ & 17 & $\begin{array}{l}\text { 4. Feedback } \\
\text { is embedded } \\
\text { in a trusting } \\
\text { relationship. }\end{array}$ & $87 \%$ & $13 \%$ & $0 \%$ & 15 \\
\hline $\begin{array}{l}\text { 5. Culture } \\
\text { emphasis is on } \\
\text { support rather } \\
\text { than blame. }\end{array}$ & $81 \%$ & $13 \%$ & $6 \%$ & 16 & & & & & \\
\hline $\begin{array}{l}\text { 6. Feedback } \\
\text { provided } \\
\text { in context } \\
\text { of trusting } \\
\text { relationships. }\end{array}$ & $88 \%$ & $6 \%$ & $6 \%$ & 17 & & & & & \\
\hline $\begin{array}{l}\text { 7. Feedback } \\
\text { recipients } \\
\text { have freedom } \\
\text { concerning how } \\
\text { they will act on } \\
\text { the feedback } \\
\text { (giving them a } \\
\text { sense of self- } \\
\text { control). }\end{array}$ & $35 \%$ & $65 \%$ & $0 \%$ & 17 & $\begin{array}{l}\text { 7. Feedback } \\
\text { recipients } \\
\text { are active } \\
\text { participants } \\
\text { in making } \\
\text { decisions about } \\
\text { how they will } \\
\text { act on the } \\
\text { feedback. }\end{array}$ & $60 \%$ & $40 \%$ & $0 \%$ & 15 \\
\hline $\begin{array}{l}\text { 8. Recipients } \\
\text { possess the } \\
\text { self-confidence } \\
\text { to allow them } \\
\text { to receive and } \\
\text { incorporate } \\
\text { feedback. }\end{array}$ & $41 \%$ & $59 \%$ & $0 \%$ & 17 & $\begin{array}{l}\text { 8. The } \\
\text { feedback } \\
\text { process } \\
\text { and culture } \\
\text { support } \\
\text { development } \\
\text { of the } \\
\text { recipient's self- } \\
\text { confidence in } \\
\text { receiving and } \\
\text { incorporating } \\
\text { feedback. }\end{array}$ & $33 \%$ & $67 \%$ & $0 \%$ & 15 \\
\hline
\end{tabular}

(Continued on next page) 
Table 1: Continued

\begin{tabular}{|c|c|c|c|c|c|c|c|c|c|}
\hline \multicolumn{5}{|c|}{ Round One } & \multicolumn{5}{|c|}{ Round Two } \\
\hline & $\begin{array}{l}\text { Essential } \\
\text { to a } \\
\text { Feedback } \\
\text { Culture }\end{array}$ & $\begin{array}{l}\text { Compatible } \\
\text { With a } \\
\text { Feedback } \\
\text { Culture }\end{array}$ & $\begin{array}{c}\text { Not } \\
\text { Important } \\
\text { to a } \\
\text { Feedback } \\
\text { Culture }\end{array}$ & $\begin{array}{c}\text { Total } \\
\mathbf{N}\end{array}$ & Revised Version & $\begin{array}{l}\text { Essential } \\
\text { to a } \\
\text { Feedback } \\
\text { Culture }\end{array}$ & $\begin{array}{c}\text { Compatible } \\
\text { With a } \\
\text { Feedback } \\
\text { Culture }\end{array}$ & $\begin{array}{c}\text { Not } \\
\text { Important } \\
\text { to a } \\
\text { Feedback } \\
\text { Culture }\end{array}$ & $\begin{array}{c}\text { Total } \\
\mathbf{N}\end{array}$ \\
\hline \multicolumn{10}{|c|}{ Essential Elements of a Feedback Culture } \\
\hline $\begin{array}{l}\text { 9. Coach for } \\
\text { performance } \\
\text { change- } \\
\text { The recipient } \\
\text { engages in } \\
\text { "change talk" } \\
\text { and develops an } \\
\text { action plan that } \\
\text { he/she feels is } \\
\text { achievable. }\end{array}$ & $47 \%$ & $53 \%$ & $0 \%$ & 17 & $\begin{array}{l}\text { 9. The } \\
\text { feedback } \\
\text { provider } \\
\text { facilitates the } \\
\text { recipient's } \\
\text { engagement } \\
\text { in identifying } \\
\text { areas for } \\
\text { change and } \\
\text { developing } \\
\text { an action } \\
\text { plan that he/ } \\
\text { she feels is } \\
\text { achievable. }\end{array}$ & $73 \%$ & $27 \%$ & $0 \%$ & 15 \\
\hline $\begin{array}{l}\text { 10. Feedback } \\
\text { is provided } \\
\text { to enhance } \\
\text { professional } \\
\text { growth. }\end{array}$ & $88 \%$ & $12 \%$ & $0 \%$ & 17 & & & & & \\
\hline $\begin{array}{l}\text { 11. A } \\
\text { continuous } \\
\text { practice } \\
\text { improvement } \\
\text { environment } \\
\text { exists. }\end{array}$ & $35 \%$ & $65 \%$ & $0 \%$ & 17 & $\begin{array}{l}\text { 11. A } \\
\text { continuous } \\
\text { practice } \\
\text { improvement } \\
\text { environment } \\
\text { exists. }\end{array}$ & $40 \%$ & $60 \%$ & $0 \%$ & 15 \\
\hline $\begin{array}{l}\text { 12. Peers and } \\
\text { faculty practice } \\
\text { feedback in } \\
\text { both high- and } \\
\text { low-stakes } \\
\text { environments. }\end{array}$ & $47 \%$ & $40 \%$ & $13 \%$ & 15 & $\begin{array}{l}\text { 12. Peers and } \\
\text { faculty give } \\
\text { feedback in } \\
\text { both high- and } \\
\text { low-stakes } \\
\text { environments. }\end{array}$ & $33 \%$ & $53 \%$ & $13 \%$ & 15 \\
\hline $\begin{array}{l}\text { 13. Clear } \\
\text { performance } \\
\text { measurements } \\
\text { exist. }\end{array}$ & $18 \%$ & $76 \%$ & $6 \%$ & 17 & & & & & \\
\hline $\begin{array}{l}14 \text {. The } \\
\text { recipient is } \\
\text { clear about } \\
\text { what the data } \\
\text { mean for his/ } \\
\text { her practice } \\
\text { and the } \\
\text { opportunities } \\
\text { for change } \\
\text { suggested by } \\
\text { the data. }\end{array}$ & $73 \%$ & $27 \%$ & $0 \%$ & 15 & $\begin{array}{l}\text { 14. The } \\
\text { recipient is } \\
\text { clear about } \\
\text { what the } \\
\text { feedback } \\
\text { means for } \\
\text { his/her } \\
\text { continued } \\
\text { development } \\
\text { and the } \\
\text { opportunities } \\
\text { for change } \\
\text { suggested by } \\
\text { the feedback. }\end{array}$ & $100 \%$ & $0 \%$ & $0 \%$ & 15 \\
\hline
\end{tabular}

(Continued on next page) 
Table 1: Continued

\begin{tabular}{|c|c|c|c|c|c|c|c|c|c|}
\hline \multicolumn{5}{|c|}{ Round One } & \multicolumn{5}{|c|}{ Round Two } \\
\hline & $\begin{array}{c}\text { Essential } \\
\text { to a } \\
\text { Feedback } \\
\text { Culture }\end{array}$ & $\begin{array}{c}\text { Compatible } \\
\text { With a } \\
\text { Feedback } \\
\text { Culture }\end{array}$ & $\begin{array}{c}\text { Not } \\
\text { Important } \\
\text { to a } \\
\text { Feedback } \\
\text { Culture }\end{array}$ & $\begin{array}{c}\text { Total } \\
\mathbf{N}\end{array}$ & Revised Version & $\begin{array}{c}\text { Essential } \\
\text { to a } \\
\text { Feedback } \\
\text { Culture }\end{array}$ & $\begin{array}{c}\text { Compatible } \\
\text { With a } \\
\text { Feedback } \\
\text { Culture }\end{array}$ & $\begin{array}{c}\text { Not } \\
\text { Important } \\
\text { to a } \\
\text { Feedback } \\
\text { Culture }\end{array}$ & $\begin{array}{c}\text { Total } \\
\mathbf{N}\end{array}$ \\
\hline \multicolumn{10}{|c|}{ Essential Elements of a Feedback Culture } \\
\hline $\begin{array}{l}\text { 15. Feedback } \\
\text { is embedded } \\
\text { implicitly and } \\
\text { explicitly in all } \\
\text { activities and in } \\
\text { which learners } \\
\text { give feedback to } \\
\text { teachers as well } \\
\text { as teachers to } \\
\text { learners. }\end{array}$ & $63 \%$ & $38 \%$ & $0 \%$ & 16 & $\begin{array}{l}\text { 15. Learners } \\
\text { give feedback } \\
\text { to teachers } \\
\text { as well as } \\
\text { teachers to } \\
\text { learners (ie, } \\
\text { feedback is } \\
\text { bidirectional). }\end{array}$ & $73 \%$ & $27 \%$ & $0 \%$ & 15 \\
\hline \multicolumn{10}{|c|}{ Ways to Operationalize a Feedback Culture } \\
\hline $\begin{array}{l}\text { 1. Direct } \\
\text { observation of } \\
\text { performance } \\
\text { is used to } \\
\text { generate } \\
\text { feedback. }\end{array}$ & $75 \%$ & $25 \%$ & $0 \%$ & 16 & $\begin{array}{l}\text { 1. Givers of } \\
\text { feedback } \\
\text { routinely } \\
\text { use direct } \\
\text { observation } \\
\text { as a source } \\
\text { of their } \\
\text { feedback. } \\
\end{array}$ & $93 \%$ & $7 \%$ & $0 \%$ & 15 \\
\hline $\begin{array}{l}\text { 2. Daily triggers } \\
\text { for feedback } \\
\text { to reinforce } \\
\text { feedback as a } \\
\text { habit, such as } \\
\text { building it into } \\
\text { care transitions } \\
\text { or other } \\
\text { educational } \\
\text { routines. }\end{array}$ & $38 \%$ & $63 \%$ & $0 \%$ & 16 & $\begin{array}{l}\text { 2. Daily } \\
\text { triggers for } \\
\text { feedback } \\
\text { are used to } \\
\text { reinforce } \\
\text { feedback as a } \\
\text { habit. }\end{array}$ & $33 \%$ & $67 \%$ & $0 \%$ & 15 \\
\hline $\begin{array}{l}\text { 3. Time } \\
\text { provided to } \\
\text { review and } \\
\text { clarify feedback } \\
\text { results with } \\
\text { others, such } \\
\text { as one's } \\
\text { supervisor/ } \\
\text { advisor/mentor. }\end{array}$ & $63 \%$ & $38 \%$ & $0 \%$ & 16 & $\begin{array}{l}\text { 3. Time is } \\
\text { provided } \\
\text { to review, } \\
\text { clarify, and } \\
\text { reflect on } \\
\text { feedback. }\end{array}$ & $87 \%$ & $13 \%$ & $0 \%$ & 15 \\
\hline $\begin{array}{l}\text { 4. Busy } \\
\text { clinicians are } \\
\text { rewarded in a } \\
\text { manner that } \\
\text { recognizes their } \\
\text { commitment } \\
\text { to a culture of } \\
\text { feedback and } \\
\text { the program } \\
\text { protects some } \\
\text { of their time to } \\
\text { engage in it. }\end{array}$ & $75 \%$ & $25 \%$ & $0 \%$ & 16 & $\begin{array}{l}\text { 4. Clinicians } \\
\text { are } \\
\text { rewarded } \\
\text { in a manner } \\
\text { that } \\
\text { recognizes } \\
\text { their } \\
\text { commitment } \\
\text { to a culture } \\
\text { of feedback. }\end{array}$ & $93 \%$ & $7 \%$ & $0 \%$ & 15 \\
\hline
\end{tabular}

(Continued on next page) 


\begin{tabular}{|c|c|c|c|c|c|c|c|c|c|}
\hline \multicolumn{5}{|c|}{ Round One } & \multicolumn{5}{|c|}{ Round Two } \\
\hline & $\begin{array}{l}\text { Essential } \\
\text { to a } \\
\text { Feedback } \\
\text { Culture }\end{array}$ & $\begin{array}{c}\text { Compatible } \\
\text { With a } \\
\text { Feedback } \\
\text { Culture }\end{array}$ & $\begin{array}{c}\text { Not } \\
\text { Important } \\
\text { to a } \\
\text { Feedback } \\
\text { Culture }\end{array}$ & $\begin{array}{c}\text { Total } \\
\mathbf{N}\end{array}$ & Revised Version & $\begin{array}{c}\text { Essential } \\
\text { to a } \\
\text { Feedback } \\
\text { Culture }\end{array}$ & $\begin{array}{c}\text { Compatible } \\
\text { With a } \\
\text { Feedback } \\
\text { Culture }\end{array}$ & \begin{tabular}{|c} 
Not \\
Important \\
to a \\
Feedback \\
Culture
\end{tabular} & $\begin{array}{c}\text { Total } \\
\mathbf{N}\end{array}$ \\
\hline \multicolumn{10}{|c|}{ Ways to Operationalize a Feedback Culture } \\
\hline $\begin{array}{l}\text { 5. Informal (or } \\
\text { "in the moment") } \\
\text { feedback is } \\
\text { encouraged. }\end{array}$ & $75 \%$ & $25 \%$ & $0 \%$ & 16 & $\begin{array}{l}\text { 5. Informal } \\
\text { or "in the } \\
\text { moment" } \\
\text { feedback is } \\
\text { encouraged. }\end{array}$ & $100 \%$ & $0 \%$ & $0 \%$ & 15 \\
\hline $\begin{array}{l}\text { 6. Reflection and } \\
\text { informed self- } \\
\text { assessment is } \\
\text { facilitated. }\end{array}$ & $75 \%$ & $25 \%$ & $0 \%$ & 16 & $\begin{array}{l}\text { 6. Reflection } \\
\text { and informed } \\
\text { self- } \\
\text { assessment is } \\
\text { facilitated. }\end{array}$ & $93 \%$ & $7 \%$ & $0 \%$ & 15 \\
\hline $\begin{array}{l}\text { 7. Feedback- } \\
\text { seeking } \\
\text { behavior is } \\
\text { encouraged. }\end{array}$ & $94 \%$ & $6 \%$ & $0 \%$ & 16 & & & & & \\
\hline $\begin{array}{l}\text { 8. Recipient- } \\
\text { initiated action } \\
\text { plans for } \\
\text { behavior change } \\
\text { are promoted. }\end{array}$ & $56 \%$ & $38 \%$ & $6 \%$ & 16 & $\begin{array}{l}\text { 8. Recipient- } \\
\text { initiated } \\
\text { action plans } \\
\text { for behavior } \\
\text { change are } \\
\text { promoted. }\end{array}$ & $73 \%$ & $27 \%$ & $0 \%$ & 15 \\
\hline $\begin{array}{l}\text { 9. Learners are } \\
\text { explicitly taught } \\
\text { how to provide } \\
\text { useful feedback. }\end{array}$ & $63 \%$ & $38 \%$ & $0 \%$ & 16 & $\begin{array}{l}\text { 9. Learners } \\
\text { are explicitly } \\
\text { taught how } \\
\text { to provide } \\
\text { useful } \\
\text { feedback. }\end{array}$ & $93 \%$ & $7 \%$ & $0 \%$ & 15 \\
\hline $\begin{array}{l}\text { 10. Faculty } \\
\text { are explicitly } \\
\text { taught how to } \\
\text { provide useful } \\
\text { feedback. }\end{array}$ & $88 \%$ & $13 \%$ & $0 \%$ & 16 & & & & & \\
\hline $\begin{array}{l}\text { 11. Skilled } \\
\text { facilitators } \\
\text { help recipients } \\
\text { interpret formal } \\
\text { (eg, multisource) } \\
\text { feedback, set } \\
\text { goals, and track } \\
\text { progress. }\end{array}$ & $44 \%$ & $50 \%$ & $6 \%$ & 16 & $\begin{array}{l}\text { 11. Train } \\
\text { recipients how } \\
\text { to interpret } \\
\text { formal (eg, } \\
\text { multisource) } \\
\text { feedback, set } \\
\text { goals, and track } \\
\text { progress. }\end{array}$ & $47 \%$ & $53 \%$ & $0 \%$ & 15 \\
\hline $\begin{array}{l}\text { 12. Regular } \\
\text { bidirectional } \\
\text { feedback } \\
\text { conversations } \\
\text { are promoted. }\end{array}$ & $63 \%$ & $38 \%$ & $0 \%$ & 16 & $\begin{array}{l}12 . \text { Regular } \\
\text { bidirectional } \\
\text { feedback } \\
\text { conversations } \\
\text { are promoted. } \\
\end{array}$ & $87 \%$ & $13 \%$ & $0 \%$ & 15 \\
\hline $\begin{array}{l}\text { 13. Reports } \\
\text { are provided } \\
\text { tying individual } \\
\text { performance } \\
\text { to bottom-line } \\
\text { departmental or } \\
\text { organizational } \\
\text { (eg, financial } \\
\text { or operational) } \\
\text { indexes. }\end{array}$ & $0 \%$ & $63 \%$ & $38 \%$ & 16 & & & & & \\
\hline
\end{tabular}

(Continued on next page) 


\begin{tabular}{|c|c|c|c|c|c|c|c|c|c|}
\hline \multicolumn{5}{|c|}{ Round One } & \multicolumn{5}{|c|}{ Round Two } \\
\hline & $\begin{array}{c}\text { Essential } \\
\text { to a } \\
\text { Feedback } \\
\text { Culture }\end{array}$ & $\begin{array}{c}\text { Compatible } \\
\text { With a } \\
\text { Feedback } \\
\text { Culture }\end{array}$ & $\begin{array}{c}\text { Not } \\
\text { Important } \\
\text { to a } \\
\text { Feedback } \\
\text { Culture }\end{array}$ & $\begin{array}{c}\text { Total } \\
\mathbf{N}\end{array}$ & Revised Version & $\begin{array}{c}\text { Essential } \\
\text { to a } \\
\text { Feedback } \\
\text { Culture }\end{array}$ & $\begin{array}{c}\text { Compatible } \\
\text { With a } \\
\text { Feedback } \\
\text { Culture }\end{array}$ & $\begin{array}{c}\text { Not } \\
\text { Important } \\
\text { to a } \\
\text { Feedback } \\
\text { Culture }\end{array}$ & $\begin{array}{c}\text { Total } \\
\mathbf{N}\end{array}$ \\
\hline \multicolumn{10}{|c|}{ Ways to Operationalize a Feedback Culture } \\
\hline $\begin{array}{l}\text { 14. Performance } \\
\text { improvements } \\
\text { following } \\
\text { feedback are } \\
\text { recognized and } \\
\text { rewarded. }\end{array}$ & $31 \%$ & $56 \%$ & $13 \%$ & 16 & $\begin{array}{l}14 . \\
\text { Performance } \\
\text { improvements } \\
\text { following } \\
\text { feedback are } \\
\text { recognized. }\end{array}$ & $47 \%$ & $53 \%$ & $0 \%$ & 15 \\
\hline & & & & & $\begin{array}{l}\text { New Item: } \\
\text { Learners } \\
\text { receive } \\
\text { training } \\
\text { on how to } \\
\text { respond } \\
\text { to both } \\
\text { positive and } \\
\text { constructive } \\
\text { feedback } \\
\text { which } \\
\text { includes } \\
\text { learning to } \\
\text { recognize } \\
\text { and manage } \\
\text { emotions } \\
\text { tied to } \\
\text { receiving } \\
\text { feedback. }\end{array}$ & $87 \%$ & $13 \%$ & $0 \%$ & 15 \\
\hline & & & & & $\begin{array}{l}\text { New Item: } \\
\text { Feedback } \\
\text { seeking } \\
\text { behavior is } \\
\text { explicitly } \\
\text { encouraged } \\
\text { during } \\
\text { orientation } \\
\text { to a new } \\
\text { organization/ } \\
\text { rotation }\end{array}$ & $53 \%$ & $47 \%$ & $0 \%$ & 15 \\
\hline
\end{tabular}

Bold text indicates met $80 \%$ consensus threshold.

comments provided during the first round and made modifications to the wording of the items based on the panelists' comments while working to maintain the original spirit of the element found in the literature. Table 1 shows the wording modifications. Based on feedback from the panelists, we also added two new items for round two: "Learners receive training on how to respond to both positive and constructive feedback which includes learning to recognize and manage emotions tied to receiving feedback," and "Feedback seeking behavior is explicitly encouraged during orientation to a new organization/rotation."

For the second round, each expert received an individualized survey, providing them with the aggregate panel rating, their own first-round rating, a collated list of open-ended comments provided during the first round, and description of any item revision. Experts used that information to rerate items using the same 3-point scale.
This study was submitted to the University of North Carolina at Chapel Hill Institutional Review Board and received a determination of not human subjects research (IRB \#182087).

\section{Results}

For round one, we received 17 responses from panelist for a response rate of $68 \%$. Three individuals declined, and we could not locate the correct email addresses for two individuals, which left 25 possible 
Table 2: Demographics of Expert Panel

\begin{tabular}{|c|c|c|}
\hline Demographics & $n$ & $\%$ \\
\hline \multicolumn{3}{|c|}{ Location } \\
\hline United States & 9 & $53 \%$ \\
\hline Canada & 4 & $24 \%$ \\
\hline Europe & 2 & $12 \%$ \\
\hline Other & 0 & $0 \%$ \\
\hline Missing & 2 & $12 \%$ \\
\hline \multicolumn{3}{|c|}{ Type of Institution } \\
\hline University & 13 & $76 \%$ \\
\hline Non-university & 2 & $12 \%$ \\
\hline Missing & 2 & $12 \%$ \\
\hline \multicolumn{3}{|c|}{ Degree } \\
\hline MD & 5 & $29 \%$ \\
\hline $\mathrm{PhD}$ & 7 & $41 \%$ \\
\hline $\mathrm{MD}, \mathrm{PhD}$ & 2 & $12 \%$ \\
\hline Something else & 1 & $6 \%$ \\
\hline Missing & 2 & $12 \%$ \\
\hline \multicolumn{3}{|c|}{ MD Specialty } \\
\hline Internal medicine & \multicolumn{2}{|c|}{3} \\
\hline Neurology & \multicolumn{2}{|c|}{1} \\
\hline Emergency medicine & \multicolumn{2}{|c|}{2} \\
\hline \multicolumn{3}{|c|}{ Work Focuses Primarily on* } \\
\hline Graduate medical education & 8 & $47 \%$ \\
\hline Undergraduate medical education & 3 & $18 \%$ \\
\hline Something else & 7 & $41 \%$ \\
\hline Missing & 2 & $12 \%$ \\
\hline
\end{tabular}

* Results not exclusive

respondents from our initial pool of 30 identified experts. For round two, 15 of the 17 experts responded, for a response rate of $88 \%$. Table 2 shows general demographics of our final expert panel. The majority $(n=13)$ worked primarily in a university setting, and about half $(\mathrm{n}=8)$ reported working in GME. Two $(n=2)$ panelists chose not to complete the demographic survey, therefore, their demographic results are missing. The panelists were diverse in their country of origin and highest academic degree.

Table 1 shows the ratings of items by the panelists for both rounds of the study. Agreement was reached for seven items in round one and to identify elements inherent to a strong feedback culture. Our process assisted us in pinpointing 17 behaviors and practices fundamental to a robust culture of feedback in a graduate medical education program. Our panelists identified universality, a supportive and trusting culture, emphasis on growth, and faculty role models as being essential elements of a feedback culture. In terms of operationalizing a feedback culture, our experts endorsed use of direct observation, time for review and reflection, rewards for commitment to a culture of feedback, encouragement of informal feedback, reflection and informed self-assessment, encouragement of feedback seeking behavior, instruction of learners and faculty on how to provide useful feedback, promotion of bidirectional feedback, and instruction for learners on responding to feedback.

Through open-ended comments, our experts also suggested that feedback must be coconstructed within the context of a meaningful and mutually respectful relationship, with the purpose being to challenge each other's thought process or actions to support growth; this idea is also grounded in the literature. ${ }^{18}$ In other words, feedback is not necessarily something that one person holds to be self-evident and then disseminates to the recipient, rather it is a joint process of developing and then acting upon what was discussed. This feedback loop, or the act of taking feedback and changing behavior, is in turn an essential part of the process and must be taken into consideration when talking about developing a feedback culture. A consideration specific to the feedback process in GME brought up by our panel experts is that in medicine, sometimes patient safety supersedes the need for coconstructed feedback and people may need to change their behavior for the sake of patient safety, regardless of whether or not they agree with the feedback. Finally, our panel identified that role modeling is an essential part of developing a culture; in the field of GME, faculty 
Table 3: Final List of Items Receiving at Least $\mathbf{8 0 \%}$ Agreement as Essential to a Culture of Feedback

\begin{tabular}{|c|c|}
\hline \multicolumn{2}{|r|}{ Item } \\
\hline \multicolumn{2}{|r|}{ Essential Elements of a Feedback Culture } \\
\hline 1 & Faculty serve as role models about how to seek, receive, and use feedback. \\
\hline 2 & Everyone receives feedback (ie, it's not voluntary). \\
\hline 3 & Feedback is embedded in a trusting relationship. \\
\hline 4 & Culture emphasis is on support rather than blame. \\
\hline 5 & Feedback provided in context of trusting relationships. \\
\hline 6 & Feedback is provided to enhance professional growth. \\
\hline 7 & $\begin{array}{l}\text { The recipient is clear about what the feedback means for his/her continued development and the opportunities for } \\
\text { change suggested by the feedback. }\end{array}$ \\
\hline \multicolumn{2}{|r|}{ Ways to Operationalize a Feedback Culture } \\
\hline 8 & Givers of feedback routinely use direct observation as a source of their feedback. \\
\hline 9 & Time is provided to review, clarify, and reflect on feedback. \\
\hline 10 & Clinicians are rewarded in a manner that recognizes their commitment to a culture of feedback. \\
\hline 11 & Informal or "in the moment" feedback is encouraged. \\
\hline 12 & Reflection and informed self-assessment is facilitated. \\
\hline 13 & Feedback seeking behavior is encouraged. \\
\hline 14 & Learners are explicitly taught how to provide useful feedback. \\
\hline 15 & Faculty are explicitly taught how to provide useful feedback. \\
\hline 16 & Regular bidirectional feedback conversations are promoted. \\
\hline 17 & $\begin{array}{l}\text { Learners receive training on how to respond to both reinforcing and constructive feedback which includes learning to } \\
\text { recognize and manage emotions tied to receiving feedback. }\end{array}$ \\
\hline
\end{tabular}

are role models. Therefore, faculty must be actively engaged in the feedback process, rather than just checking it off the list. Programs can also work toward normalizing feedback conversations in order to develop a feedback culture within their programs; they must also work toward embedding feedback implicitly and explicitly in all activities, and both learners and teachers should be encouraged to actively seek out opportunities for feedback. ${ }^{7}$

Our findings can function as a guide for family medicine educators seeking to facilitate a culture of feedback within their programs. As identified in a review of the feedback literature by Bing-You et al, few articles describe how the culture of an organization can be assessed and improved. ${ }^{19}$ Using our essential elements and ways to operationalize a feedback culture, educators can evaluate their programs and institute changes that will encourage increased feedback between learners and faculty. By providing specific steps to operationalizing a feedback culture, we are presenting a framework for faculty seeking to facilitate a positive feedback culture in their programs.

Limitations of our study include the size of our panel of experts. A panel of 17 respondents is smaller than some peer studies, though Murphy et al suggest that a sample size of 12 is reasonable for a Delphi study. ${ }^{20}$ Similarly, our participants may not be representative of experts across the entire field of graduate medical education. We generated our initial list of experts from primary authors of papers on feedback in graduate medical education but did not seek to specify if this was focused on medical school, residency programs, or specific types of residency programs. We included experts from multiple countries (Table 2), and while we believe this enriched our review it is possible that different cultures have different perspectives on feedback; therefore results may not apply to all cultures globally. Finally, while we suspect many of our findings would be useful across the medical education continuum, some may not be generalizable to undergraduate medical education. Our intent was to have a broad view of feedback culture that could be applied across multiple specialties, given family medicine residency education occurs in multiple settings. Thus, the results may not be solely applicable to family medicine training programs. Also none of the experts identified as having a family medicine or primary care specialty. Nonetheless the authors' experience 
in family medicine graduate medical education and faculty development leads us to believe it is indeed applicable to the discipline. Future studies could examine if there are differences in expert consensus related to graduate medical education specialty.

Experts theorize that a strong feedback culture fosters learning and growth in graduate medical education. Multiple studies focus on defining a culture of feedback, consider barriers to developing a culture of feedback, and discuss programs as encouraging or impeding a culture of feedback. Additionally, while tools exist to evaluate the culture of quality improvement in residency programs, no such tool exists to evaluate programmatic cultures of feedback. We address this in our study by utilizing a Delphi technique to reach agreement on essential elements of a feedback culture and ways to operationalize a feedback culture.

\section{Conclusion}

Our results enhance the current literature by defining an expert consensus on what constitutes a robust culture of feedback and providing a guide for educators on specific behaviors and practices for facilitating such a culture to foster programs conducive to learning and growth. Next steps include developing and validating an assessment tool using these identified essential elements. Using such an assessment tool will allow programs to evaluate their baseline and identify steps for improvement. This will also allow testing of hypotheses related to steps for improvement, associations between feedback culture and quality improvement culture, and impact on clinical outcomes. While evidence to support strategies for changing organizational culture is limited ${ }^{21}$ using such an assessment tool will allow programs to understand where they are on the spectrum, and then also to take steps to improve the culture of feedback for faculty and learners alike.
ACKNOWLEDGMENTS: The authors acknowledge the contributions of Alfred Reid, MA, who provided considerable guidance and assistance throughout much of the research process. The authors thank Eric Holmboe, MD, who provided guidance in the early stages of study development. The authors also thank the expert panel participants for their contribution to this work.

PRESENTATIONS: This research was presented at the 2020 STFM Annual Spring Conference (virtual)

CORRESPONDING AUTHOR: Address correspondence to Dr Cristen P. Page, UNC School of Medicine, 4030 Bondurant Hall, Campus Box 7000, Chapel Hill, NC 27599. 919-966 8933. Fax: 919-966-8623. cristy_page@med. unc.edu

\section{References}

1. Veloski J, Boex JR, Grasberger MJ, Evans A, Wolfson DB. Systematic review of the literature on assessment, feedback and physicians' clinical performance: BEME Guide No. 7. Med Teach. 2006;28(2):117-128. doi:10.1080/01421590600622665

2. de la Cruz MSD, Kopec MT, Wimsatt LA Resident Perceptions of Giving and Receiving Peer-to-Peer Feedback. J Grad Med Educ. 2015;7(2):208-213. doi:10.4300/JGMED-14-00388.1. Accessed July 16, 2018. Internet.

3. Kraut A, Yarris LM, Sargeant J. Feedback Cultivating a Positive Culture. J Grad Med Educ. 2015;7(2):262-264. doi:10.4300/JGMED-15-00103.1

4. Bing-You R, Varaklis K, Hayes V, Trowbridge R, Kemp H, McKelvy D. The Feedback Tango: An Integrative Review and Analysis of the Content of the Teacher-Learner Feedback Exchange. Acad Med. 2018;93(4):657-663 http://links.lww.com/ACADMED/A484. Accessed July 16, 2018. Internet. doi:10.1097/ ACM.0000000000001927

5. Ramani S, Könings KD, Mann KV, Pisarski EE, van der Vleuten CPM. About politeness, face, and feedback: exploring resident and faculty perceptions of how institutional feedback culture influences feedback practices. Acad Med. 2018 Sep;93(9):1348-1358. doi: 10.1097/ ACM.0000000000002193.

6. London M, Smither JW. Feedback orientation, feedback culture, and the longitudinal performance management process. Hum Resour Manage Rev. 2002;12(1):81-100. doi:10.1016/ S1053-4822(01)00043-2

7. Archer JC. State of the science in health professional education: effective feedback. Med Educ. 2010;44(1):101-108. doi:10.1111/j.13652923.2009.03546.x

8. Ramani S, Könings KD, Ginsburg S, van der Vleuten CPM. Twelve tips to promote a feedback culture with a growth mind-set: swinging the feedback pendulum from recipes to relationships. Med Teach. 2019;41(6):625-631. doi:10.1080/0142159X.2018.1432850.
9. Bodenheimer T, Ghorob A, Willard-Grace R, Grumbach K. Care UC for E in P. The 10 building blocks of primary care forming stable teamlets. Ann Fam Med. 2014;12(2):166-171. doi:10.1370/afm.1616

10. Page CP, Reid A, Coe CL, et al. Learnings from the pilot implementation of mobile medical milestones application. J Grad Med Educ. 2016;8(4):569-575. doi:10.4300/JGMED-15-00550.1

11. Page C, Reid A, Coe CL, et al. Piloting the mobile medical milestones application(M3app(C): A multi-institution evaluation. Fam Med. 2017;49(1):35-41.

12. Myerholtz L, Reid A, Baker HM, Rollins L, Page CP. Residency faculty teaching evaluation: what do faculty, residents, and program directors want? Fam Med. 2019;51(6):509-515. doi:10.22454/FamMed.2019.168353

13. Donahue KE, Reid A, Lefebvre A, Stanek M, Newton WP. Tackling the triple aim in primary care residencies: the I3 POP Collaborative. Fam Med. 2015;47(2):91-97.

14. Page CP, Reid A, Newton W. Variation in Residency Triple aim measures: residencies' clinical signatures. J Grad Med Educ. 2018;(October):548-552. doi:10.4300/JGME-D-17-00470.1

15. MacColl Center for Health Care Innovation. Patient Centered Medical Home Assessment. http://www.safetynetmedicalhome.org/resources-tools/assessment. Published 2014. Accessed November 12, 2019

16. Bradley EH, Brewster AL, Fosburgh H, Cherlin EJ, Curry LA. Development and psychometric properties of a scale to measure hospital organizational culture for cardiovascular care. Circ Cardiovasc Qual Outcomes. 2017;10(3):1-8. doi:10.1161/CIRCOUTCOMES.116.003422

17. Humphrey-Murto S, Varpio L, Gonsalves C, Wood TJ. Using consensus group methods such as Delphi and Nominal Group in medical education research. Med Teach. 2017;39(1):14-19. doi:10.1080/0142159X.2017.1245856

18. Ajjawi R, Regehr G. When I say ... feedback. Med Educ. 2019;53(7):652-654. doi:10.1111/ medu. 13746

19. Bing-You R, Hayes V, Varaklis K, Trowbridge $\mathrm{R}, \mathrm{Kemp} \mathrm{H}, \mathrm{McKelvy}$ D. Feedback for learners in medical education: what is known? A scoping review. Acad Med. 2017;92(9):1346-1354. doi:10.1097/ACM.0000000000001578

20. Murphy MK, Black NA, Lamping DL, McKee CM, Sanderson CF, Askham J, Marteau T. Consensus development methods, and their use in clinical guideline development. Health Technol Assess. 1998;2(3):i-iv, 1-88

21. Parmelli E, Flodgren G, Beyer F, Baillie N, Schaafsma ME, Eccles MP. The effectiveness of strategies to change organisational culture to improve healthcare performance: a systematic review. Implement Sci. 2011;6(1):33. doi:10.1186/1748-5908-6-33 